Penultimate draft. For published version see:

https://www.tandfonline.com/doi/full/10.1080/0020174X.2021.1976270

Błażej Skrzypulec

Institute of Philosophy, Jagiellonian University, ul. Grodzka 52, 31-044 Kraków, Poland E-mail: blazej.skrzypulec@uj.edu.pl

ORCID: 0000-0003-2780-5477

\begin{abstract}
Philosophers of perception often claim that usual perceptual experiences not only present particulars but also phenomenally present them as particulars. Nevertheless, despite the initial plausibility of this thesis, it is not clear what exactly it means to say that particularity is phenomenally presented. The paper aims to provide a deeper analysis of the claim that perceptual experiences phenomenally present objects as particulars. In doing so, I distinguish two theses regarding phenomenally presented particularity: Generic Particularity and Specific Particularity. According to the first thesis, vision phenomenally presents particularity of objects, understood as a general characteristic that may be shared by many entities. The second thesis states that vision phenomenally presents particularity of objects, understood as an individual characteristic unique to each particular. I argue that, relying on knowledge concerning the functioning of perceptual mechanisms, vision does not phenomenally present generic particularity but it has certain abilities for presenting specific particularity.
\end{abstract}

Keywords: perception; particularity; phenomenal character; representational content; attention 


\section{Perceptual experiences of particularity}

It is commonly believed that our perceptual experiences are largely about particulars (e.g., Jeshion 2010; Mehta 2014; Schellenberg 2016; Soteriou 2000). First, it seems plausible that ordinary perceptual experiences are caused by particular things, for instance tables, birds, and trees. Furthermore, perceptual experiences seem to provide knowledge about perceived particulars and allow us to form singular thoughts about their properties (e.g., Brewer 2015; Martin 2002a; Nanay 2012). In addition, a stronger thesis is often proposed that perceptual experiences not only present particulars but also phenomenally present them as particulars (e.g., Mehta 2014; Montague 2011; Schellenberg 2016). In other words, when perceiving a particular item, we are not only perceptually related to a particular, but its particularity is usually somehow reflected in the phenomenal character of an experience.

For instance, Mehta (2014), in discussing the individuation of phenomenal character, notices that introspection suggests that perceptual experiences present objects not as being some objects with certain properties, but as these particular objects. This aspect of perceptual experience is explicitly recognized as "phenomenological particularity" by Schellenberg (2016), who believes that it is uncontroversial that perceptual, or at least visual, phenomenology presents objects as particulars. Similarly, Gomes and French (2019) also use the term "phenomenological particularity" to express the idea that the particularity of experienced objects is reflected in phenomenology.

Furthermore, the conviction that the particularity of objects is phenomenally presented in perceptual experiences plays a role in justifying certain positions in current philosophical debates. Most importantly, it has relevance for discussions concerning the particularity and generality of perceptual content (e.g., Dokic 1998; Schellenberg 2010; Schroer 2014; Soteriou 2000). It is commonly claimed that the phenomenal character of experiences is closely 
correlated with their representational content (see Byrne 2001; Green 2016; Macpherson 2006). If this is the case, then the fact that particularity is phenomenally presented makes it more plausible that representational content should not be described merely in general terms either (e.g., in a form of quantified general proposition).

Nevertheless, despite the fact that it seems plausible to accept that objects are phenomenally presented as particulars and that this thesis is not without significance for the contemporary philosophy of perception, it is not clear what exactly it means to say that particularity is phenomenally presented. Usually, this aspect of perceptual experiences is simply assumed by relying on intuition, without any attempt to provide proper justification of its validity or explication of the notion of 'particularity'.

In this paper, I aim to provide a deeper analysis of the claim that perceptual experiences phenomenally present objects as particulars. In doing so, I distinguish two variants of phenomenally presented particularity: 'generic particularity' and 'specific particularity.' Relying on this distinction I formulate two theses: Generic Particularity and Specific Particularity. According to the Generic Particularity thesis, vision phenomenally presents particularity of objects, understood as a general characteristic that may be shared by many entities. The Specific Particularity thesis states that vision phenomenally presents particularity of objects, understood as an individual characteristic unique to each particular. I argue that, relying on knowledge concerning the functioning of perceptual mechanisms, the Specific Particularity thesis is justified in case of human visual experiences, but that there is no such justification for the Generic Particularity thesis. While investigating whether perception phenomenally presents the particularity of objects, I do not assume any specific theory of perception. What I consider is the general idea that the way we perceptually experience objects is such it is plausible to characterize it by appealing to the notion of particularity. This general idea can be specified by a Russellian representationalist as a claim 
that vision presents particularity by presenting objects as instantiating certain properties, while a Freagean representationalist may claim that objects are presented under the particular mode of presentation, and a direct realist can postulate that a perceptual relation between an object and a subject is such that it reveals the particular status of objects.

In the first section, I introduce a distinction between generic and specific particularity. ${ }^{1}$ Subsequently (in Section 2), I explicate the notion of generic particularity and argue that vision does not present particularity of objects in the sense of generic particularity. It is only possible to justify weaker theses that vision does not present objects as having characteristics which are incompatible with generic particularity or that vision presents objects as having characteristics which imply generic particularity. In Sections 3 and 4, I investigate visual presentations of specific particularity. In Section 3, the notion of demonstrative phenomenology is introduced, and serves as a basis for showing that human vision presents specific particularity. Finally, in Section 4, relying on the content of previous sections, I argue that human vision has a limited ability to phenomenally present the specific particularity of several objects at once.

\section{Two variants of phenomenal particularity}

The thesis that vision phenomenally presents objects as particulars can be understood in two main ways. It is not necessarily the case that all entities are particulars; some may be nonparticular entities such as universals (see Armstrong 1978ab; Loux 1978; Lowe 2006 for classic sources). From this perspective, particularity is a general characteristic that can be

\footnotetext{
${ }^{1}$ I adopt this terminology form Gomes and French (2019). An important difference is that, according to Gomes and French, any experience with phenomenal character that can be described in general terms, for instance, as an experience as of an apple or as of something red, exhibits generic particularity. In contrast, I believe that this requires further argument (see section 2).
} 
shared by many entities, while other entities may lack it. For instance, an object $A$ may be numerically distinct from an object $B$, but they both may possess the characteristic of being particulars ${ }^{2}$. Further, I name this understanding of particularity 'generic particularity' and I consider the following Generic Particularity thesis:

(Generic Particularity) Vision phenomenally presents, under standard conditions, particularity of objects, where particularity is understood as a general characteristic that may be shared by many entities.

In considering whether generic particularity is phenomenally presented, I restrict myself to investigating visual experiences. First, human vision is the main point of focus in earlier works concerning relations between phenomenology and particularity (e.g., Campbell 2002; Martin 2002a; Schellenberg 2016). Second, including other modalities goes beyond the scope of a single paper, as it not clear that all modalities present objects, and rules governing perceptual organization may be significantly different among the senses (see O'Callaghan 2008; Martin 1992; Skrzypulec 2019). Furthermore, I am concerned only with the phenomenal presentations of objects, and the results presented cannot be automatically applied to debates regarding, for instance, the particular status of visually presented properties (see Almäng 2013; Nanay 2012). The remark about standard conditions is introduced to allow for cases of misperception regarding particularity. I do not exclude the possibility that under some circumstances vision may present particular objects as not particular or nonparticular entities as particulars. However, in this paper I do not assess whether such mistakes

\footnotetext{
2 I use the term 'characteristic' to remain natural regarding the specific metaphysical explanation of two entities potentially being both particulars. For instance, one may propose that particularity is a universal property possessed by many entities, or being a particular consists in exemplifying a certain ontological category, or that being particular is a primitive fact which does not require a further specification.
} 
actually occur or what the causes of such mistakes might be. Assessing whether the Generic Particularity thesis is justified requires determining what particularity, understood as a general characteristic, means. These investigations are conducted in section 2 where I argue against the Generic Particularity thesis.

The statement that the particularity of objects is phenomenally presented can be also understood in a different way. According to this version, objects are experienced as unique, individual beings. This means that if an object is phenomenally presented it is presented as this individual object $A$ (which is numerically distinct from objects $B$, $C$, etc.). I call this understanding of particularity 'specific particularity' and investigate the following Specific Particularity thesis:

(Specific Particularity) Vision phenomenally presents, under standard conditions, particularity of objects, where particularity is understood as a unique, individual characteristic.

According to the Specific Particularity thesis, visual perception phenomenally presents particularity by presenting an object as having a unique characteristic that is not shared with other objects $^{3}$. In sections 3 and 4, I argue that the Specific Particularity thesis holds in the case of human vision, but the scope of its validity is restricted.

My results have two consequences for the current debates in the philosophy of perception. The first one concerns the debate between representationalism and naïve realism. Representationalist theories of perception tend to be associated with the narrow notion of

\footnotetext{
${ }^{3}$ Analogously, as in the case of generic particularity, I do not assume any specific metaphysical structure in virtue of which specific particularity is realized. For example, it may be postulated that an object has specific particularity in virtue of instantiating a particularized trope-property or due to being partially constituted by a particular element such as 'bare substratum' or 'thisness.'
} 
phenomenology, according to which, experiences are phenomenally the same only if the phenomenology of one experience cannot be, even in optimal conditions, introspectively distinguished from the phenomenology of the second experience (see Byrne 2001, Schellenberg 2010, Tye 1995). One reason is that perception does not seem to be able to differentiate between distinct particulars with the same perceptible characteristics, for instance the phenomenology of experiencing a red ball $A$ may be exactly the same as when a red ball $B$ is perceived. On the other hand, naïve realist approaches often include a wide notion of phenomenology in which phenomenal character is constituted by perceived particulars, so subjectively indistinguishable experiences can have distinct phenomenal characters (see Brewer 2011, Logue 2012, Martin 2002a). This division may suggest that representationalist theories using a narrow notion of phenomenology are inconsistent with a claim that specific particularity is phenomenally presented. I show that it is not the case, as to some degree, specific particularity can be included within the narrow phenomenology.

Second, the truth of the Specific Particularity thesis is important if one wants to justify the particular notion of perceptual content relying on the particularity of phenomenal character (see Schellenberg 2016; Martin 2002b). The particularity described by the Generic Particularity thesis can be accommodated by general notions of representational content as content determined by such phenomenal character may be characterized by using general quantified formulas. However, if phenomenal character satisfies the Specific Particularity thesis, then the corresponding representational content would contain an individual, unrepeatable element. In consequence, an adequate characterization of such content would involve a singular term and so would not take the form of a general proposition.

\section{Generic particularity}


Though philosophical works concerning perception frequently offer remarks to the effect that perception presents particulars, or that perception presents some entities as particulars, it is rarely explicitly stated what generic particularity is believed to be. Sometimes, being particular is associated with being mind-independent, or with having the ability to causally influence perceptual systems (Schellenberg 2016, 4). However, such characteristics do not seem crucial for generic particularity.

First, the notion of mind-independence seems to be orthogonal to the notion of generic particularity. For instance, physical properties, such as having a certain electrical charge, are often characterized as universals and not as particulars, though they are treated as mindindependent (see MacBride 1998). On the other hand, in classic sense-data theories of perception, sense-data are interpreted as mind-dependent particulars. Similarly, it is not without controversies that lack of particularity entails lack of causal powers. In particular, according to classic Armstrong's theory, properties are universals yet possess casual powers, or are even individualized by their abilities to enter causal relationships (see Armstrong 1978, 47-50). Furthermore, even if one accepts that causal powers can be attributed only to particularized instances of properties, a reverse problem is still present, namely that it is not obvious that all particulars have causal powers, because while it is common to treat certain abstract entities, such as numbers (see Melia 2008; Giberman 2016), as particulars, it is debatable that they can enter casual relations. Hence, characteristics such as mindindependence or having causal powers do not seem to be identical to the particularity, rather they are associated with some types of particulars. Even if particulars presented in perceptual experiences are mind-impendent and have causal powers, phenomenal experiences of these characteristics are not phenomenal experiences of particularity, but of other characteristics possessed by perceptible particulars. 
Below, in order to focus on the characteristic of particularity itself, I consider ideas developed in the field of analytic metaphysics, and in this context analyze whether generic particularity is phenomenally experienced. I discuss three definitions of particularity: (a) as an inability for multiple instantiation, (b) as a lack of multiple localization, and (c) as independence of indiscernibility and identity. It is argued that such modal characteristics are unlikely to be phenomenally presented.

\subsection{Particularity as an inability for multiple instantiation}

As suggested above, the concept of particularity is crucial in metaphysical debates regarding the problem of universals, as universals are exactly those entities that should be distinguished from particular entities. Probably the most influential way to discern between universals and particulars is to postulate that only the former can be wholly and simultaneously multiply instantiated (e.g., Lowe 2006, 114-115; Moreland 2001, 3-4). For instance, the property of redness may be at the same moment instantiated by more than one object (e.g., by a red ball and a red car). In addition, it does not seem that redness is composed of two parts, one instantiated by a ball and a second instantiated by a car. Instead, redness is fully instantiated by each of these objects. On the other hand, a particular such as a table seems to be a subject of properties and not a property itself, so it is not instantiated by anything, and in consequence, cannot be multiply instantiated. Of course, to interpret particularity in terms of instantiation in the perceptual context, one has to endorse a potentially controversial assumption that it is possible to perceptually experience instantiation. Here, I do not want to accept this assumption or reject it; I merely adopt it conditionally in order to discuss the considered understanding of particularity. 
Given the remarks above, it may be proposed that particularity, understood as a general characteristic, consists in an inability for multiple instantiation. If such notion of particularity is adopted, then the Generic Particularity thesis states that vision phenomenally presents objects as being incapable of multiple instantiation ${ }^{4}$. However, it is unlikely that such modal and negative characteristic can be ascribed to objects by human vision. It seems that only a weaker thesis can be plausibly justified: that vision does not present objects as having characteristics which are incompatible with particularity, i.e. with an inability to be multiply instantiated. It is so because vision, at least under normal conditions, does not present objects as having a characteristic of multiple instantiation. In consequence, by not presenting objects as being multiply instantiated vision does not present them as having a characteristic which is incompatible with the inability for being multiply instantiated. This conviction is justified by the fact that in the case of usual visual states, objects are presented not as instantiated properties but as subjects which possess features and unify them into perceptual units.

In the philosophical literature, there is no agreement regarding the proper theory of instantiation. For instance, while instantiation is often interpreted as a relation, Armstrong has characterized it as a "non-relational tie" (Armstrong 1997, 30). Furthermore, it is debatable whether instantiation is a primitive relation or can be analyzed in terms of identity (see Baxter 2001; Brown 2017) or localization (see Cowling 2014). Nevertheless, despite these differences, one of the main intuitions regarding instantiation is that it is a link that combines an entity with its property (see Orilia and Paoletti 2020 for a review). In consequence, when instantiation occurs, there is a subject which instantiates a property, and a property which is instantiated by the given subject-entity. For instance, if instantiation combines Socrates with

\footnotetext{
${ }^{4}$ In this paper, I merely consider whether the particularity of objects is phenomenally presented; I do not address the issue of the phenomenal particularity of experienced properties. It seems possible that particularity of objects is not phenomenally presented, but particularity of properties is presented (or vice versa).
} 
"being wise" and "being a Greek," then both these properties are instantiated by the common subject: Socrates.

By applying this idea to the perceptual context, we may ask whether vision presents objects (a) as subjects instantiating properties or (b) as properties instantiated by some entities. If the first option is true, then objects are not experienced as something instantiated. In consequence, they are also not experienced as something multiply instantiated. As a result, because being multiply instantiated is incompatible with particularity understood as an inability to be multiply instantiated, objects are not visually presented as having a characteristic incompatible with particularity.

In fact, it seems that it is far more plausible to claim that objects are visually presented as subjects instantiating properties and not as instantiated properties, because an object is visually experienced as a perceptual unit to which some properties are attributed. For example, an object may be experienced as a figure that is a small, red square. In virtue of visually attributed properties, such units are differentiated from the ground (Qiu and von der Heydt 2005), may be tracked while they move (Scholl 2007), can be perceived as parts of a larger entity (Xu and Singh 2002), and may be categorized as exemplars of categories of objects (Biederman 1987). The experiential subject-status of objects suggests that vision does not present objects as having a characteristic incompatible with particularity, understood as an inability to be multiply instantiated. However, there is no evidence that vision presents objects as having a modal characteristic of being unable to possess multiple instantiations.

\subsection{Particularity as a lack of multiple localization}

Characterizing particulars as incapable of multiple instantiation is not the only influential approach to defining particularity. Alternatively, the notion of particularity is 
explicated in terms of an ability to be wholly, simultaneously, multiply localized at disjoint places (e.g., Daly 1994; Heil 2003, 132-136). A particular object, such as a table, does not seem to have an ability to be wholly multiply located at a single moment. The only multiple localization it can achieve is such that it can have proper parts, each with distinct localization. In contrast, a universal property like redness can be wholly present at many disjoint localizations.

Nevertheless, inability to be multiply localized is also a modal notion and is unlikely to be phenomenally presented by human vision. Once again, only a weaker thesis is justified - that vision, under normal circumstances, does not present objects as having characteristics incompatible with an inability to be multiply localized. In other words, vision does not present a single object as simultaneously located in disjoint places. It is so because spatial separation is the strongest visual cue for recognizing the distinctiveness of objects (see Palmer and Rock 1994). If an object $A$ is visually experienced as positioned at place $P_{1}$, and an object $B$ is visually experienced as positioned at disjoint place $P_{2}$, then $A$ and $B$ may be experienced as two separate objects, or as two distinct parts of a larger object (as in cases of perceptual grouping or visual completion, Kubovy and Wagemans 1995), but there are no cases in which $A$ is experienced as numerically identical to $B$. One may ask whether experiences involving reflections, like when both an object and its mirror-image are experienced, constitute a case in which an object is phenomenally presented as wholly positioned in two disjoint places. Experiences involving reflections can be interpreted in two general ways. First, it may be claimed that at the level of perceptual processing there is no ability to distinguish actual objects from mirror-images as such a distinction is established in virtue of some post-perceptual mechanisms. If this is the case, then when perceiving an object and its mirror-image we simply, and inaccurately, experience two distinct objects. A second option is to claim that the human visual system is able to present the property of being a 
mirror-image. However, in this interpretation too there is no justification for claiming that while perceiving an object and its mirror-image we experience a single object wholly present in two places. What happens in such a situation is a veridical experience of a single object positioned in some place and having a reflection in a mirror positioned in a distinct location.

\subsection{Particularity as independence of indiscernibility and identity}

While the characterization of particularity in terms of the potential for multiple instantiation or localization has gained significant popularity, it is not universally accepted. Some authors believe that there are plausible examples of universals that do not have the possibility of multiple instantiation (e.g., the property of having the highest electrical charge in the universe, MacBride 1998) and of particulars that have such an ability (e.g., a particularized property that travels through time and meets itself in the past, Ehring 2004).

Because of such problems, an alternative distinction has been proposed that distinguishes universals from particulars by virtue of a relation between indiscernibility and numeric identity (Ehring 2004; 2011). It is proposed that in the case of universals, having the same intrinsic (i.e., non-relational) properties is sufficient for numeric identity. According to this approach, assuming that color-properties are universals, if a ball has a certain shade of red and a car has an indiscernible shade of red, then the color-property of a ball is numerically identical with the color-property of a car. On the other hand, the analogous inference does not occur in the case of particulars. For instance, it is possible that there are multiple numerically distinct tables all with the same non-relational properties (e.g., color, shape, size, etc.).

If such an understanding of particularity is adopted, then the Generic Particularity thesis is true if vision presents objects in a manner such that it is possible for them to have numerically distinct, indiscernible duplicates. However, once again, it is a modal 
characteristic which is unlikely to be visually experienced. On the other hand, analogous to the case of previous notions of particularity, it is justified to claim that vision, under normal conditions, does not present objects as having characteristics incompatible with particularity. Furthermore, it is likely that if particularity is understood as a possibility of having indiscernible duplicates a stronger claim can be accepted, namely that vision sometimes presents objects as having characteristics which imply particularity. More specifically, vision can present an object $a$ and an object $b$ as distinct while presenting them as possessing the same nonrelational properties. If objects are distinct while indiscernible, then they actually have indiscernible distinct duplicates, and so it is possible for them to have such duplicates.

In fact, it is easy to obtain an experience in which objects are visually presented as distinct while being presented as sharing all intrinsic properties and differing only in localization. For instance, a person may have an experience as of two uniformly black circles with the same diameter and positioned in proximal locations. In contrast, it is hardly possible to find visual experiences presenting objects as distinct while presenting them as co-located and sharing all intrinsic properties such as color, shape, or size. Such objects would completely overlap and would not be visually experienced as distinct individuals. Thus, it is crucial to consider whether location-related properties are visually presented as relational or intrinsic. If such properties are relational, then there are plenty of examples in which objects are visually presented as distinct while being presented as indiscernible, as there are many cases in which localization is the only characteristic that differentiates between visually experienced objects.

According to the usual psychological considerations, space is visually represented in two ways: egocentric and allocentric (see Klatzky 1998 for a review). In the case of egocentric representations, perceived objects are presented as positioned in some direction and at some distance from the observer. For example, a spatial distinction between two black 
circles may consist of experiencing one of them as positioned on the right of the observer and the second one on the left. In contrast, in allocentric representations, objects are spatially organized not by their relation to the observer but by relations between themselves. For instance, the positions of black circles may be experienced as distinct because one circle is experienced as being to the left of the other. Despite differences between egocentric and allocentric forms of visual spatial representations, it is clear that both have an important relational aspect. In the case of egocentric representations, having a spatial location means being positioned in relation to a perceiving subject, and in the case of allocentric representations, having a location concerns being positioned relative to other objects. Because of this, it is plausible to interpret visual properties concerning localization as relational properties. In consequence, there are a number of visual experiences in which objects are experienced as numerically distinct (due to different localizations) but indiscernible (because they possess same intrinsic properties such as color, size, shape, etc.).

Nevertheless, even if vision sometimes presents objects as having characteristics which imply particularity, it does not follow that it presents objects as having particularity. In general, it is far from obvious that vision, when presenting an object as being $F$, also presents this object as having all properties logically entitled by $F$. For instance, having length $X$ implies not having length $X+1$, not having length $X+100$, being shorter than an object with length $2 X$, etc. However, it seems implausible to state that when vision phenomenally presents something as having length $X$ it also phenomenally presents a given object as having an infinite number of negative and comparative properties.

Overall, while characteristics such as mind-independentness and causal efficacy are sometimes considered in philosophical investigations about phenomenal particularity, they are merely related and not identical to the generic particularity. More accurate notions of generic particularity have been developed within analytic metaphysics. However, these 
characteristics, such as inability to be multiply instantiated, inability to be multiply localized, and the possibility of having indiscernible duplicates are modal characteristics, and are unlikely to be phenomenally presented by human vision. In consequence, there is no strong justification for the Generic Particularity thesis. Only weaker these can be plausibly accepted - that vision, at least under normal circumstances-does not present objects as having characteristics incompatible with particularity or that vision sometimes presents objects as having characteristics which imply particularity. These results show that there are some constraints on what can be phenomenally presented which are related to the generic particularity. However, the presence of such constraints does not imply that generic particularity is phenomenally presented, but merely that some characteristics, inconsistent with generic particularity, are not presented.

\section{Specific particularity}

While it is unlikely that human vision phenomenally presents the generic particularity of objects, the status of the specific particularity thesis is still unclear. According to this thesis, vision presents particularity of objects understood as a unique, individual characteristic. The intuitive problem with this idea is that there are possible numerically distinct objects that, despite their distinctiveness, are phenomenally experienced in exactly the same way (see Hill 2019). For instance, if an object $A$ and an object $B$ are the same in all respects related to the reflectance of light, such as hue, size, shape, and smoothness of surface, and are presented in the same context, then the visual experience of $A$ would be phenomenally exactly the same as the experience of $B$. In consequence, it seems that the visual system is not able to phenomenally present objects as individual particulars. Nevertheless, the above reasoning is only able to show that human vision cannot phenomenally present the specific particularity of 
every object in every circumstance. However, it still may be the case that the visual system has some more restricted abilities in presenting specific particularity. Below, I argue for such a position, relying on psychological models describing human object perception.

My argumentation in favor of the specific particularity thesis consists of four steps. First, I introduce the notion of 'demonstrative phenomenology' which captures the fact that in perceptual experiences we are presented with this object. Second, I argue that demonstrative phenomenology is a form of perceptual phenomenology. This is to ensure that demonstrative phenomenology is not some post-perceptual phenomenon, and so specific particularity is not perceptually experienced. The two above points are presented in section 3.1. Further, I propose that that demonstrative phenomenology occurs due to the application of 'individuation devices' described in psychological models of object perception. Finally, I argue that when several objects are represented by individuation devices, each is associated with distinct demonstrative phenomenology; therefore, specific particularity is experienced. If demonstrative phenomenology were the same for each represented object, referring to it would not allow justifying the specific particularity thesis. These two stages are described in section 3.2.

The proposed position is consistent with an intuition that phenomenal differences between experiences are, in principle, introspectively accessible to a subject. The accommodation of this intuition constitutes a link between my considerations and the debate about narrower and broader characterizations of phenomenology. In the narrow notion, phenomenology is described in terms of the way surroundings appear to a subject. The characterization of broad phenomenology goes beyond what appears to a subject, and also specifies an entity which appears to a subject in a certain way. For instance, Beck (2019) characterizes narrow phenomenology as composed of 'appearance properties,' like being 
appeared in a bluish way, while broad phenomenology is composed of 'item-in-appearance properties' such as being a subject to whom x appears bluish ${ }^{5}$.

It is plausible to claim that in the case of narrow phenomenology all phenomenal differences between experiences are, in principle, introspectively accessible to a subject. On the other hand, it is less obvious in the case of broad phenomenology, as there may be differences which concern only the experienced entities, and not the way in which these entities appear to a subject. For instance, a difference between being a subject to whom $x$ appears bluish and being a subject to whom y appears bluish may not be detectable by introspecting the phenomenology of perceptual experience, no matter the circumstances.

My account shows that at least some differences in phenomenal presentations of specific particularity are, in principle, introspectively accessible. In consequence, my argument shows that one can maintain a thesis that specific particularity is phenomenally presented even if one understands phenomenology according to the narrow notion.

\subsection{Phenomenal demonstratives and perceptual phenomenology}

In developing an account of phenomenal specific particularity, I rely on the idea that human visual experiences have a phenomenal demonstrative aspect (e.g., Mehta 2014; Montague 2011; Soteriou 2000). According to this idea, when we perceive an object, in particular by focusing attention on it, we not only experience that there is an object with a feature $F$ and positioned in place $P$, we also experience that those characteristic are possessed by this object to which we attend. In this context, two questions should be asked: first, whether such demonstrative phenomenal aspect belongs to the phenomenology of perceptual states; and

\footnotetext{
${ }^{5}$ Gomes and French (2019) make an analogous distinction by developing Martin's (2002a) notions of narrower phenomenal character and broader phenomenal nature.
} 
second, whether it is the case that such phenomenal aspect can be plausibly interpreted as presenting the specific particularity of the attended object. I confront the first of these questions in this section and the second in section 3.2.

It seems intuitive that focusing attention on an object introduces an additional, demonstrative phenomenal aspect to the experience. However, it is not obvious whether this demonstrative phenomenal aspect belongs to the perceptual phenomenology. On the one hand, it is well established that attention modifies visual phenomenology (Carrasco and Barbot 2019). For instance, the color of an attended object may look more saturated (Fuller and Carrasco 2006), an attended object may seem closer to the observer (Green 2016), and its features may be represented in a more determined way (Prinzmetal et al. 1998). Furthermore, some properties, such as complex shapes, may be experienced only attentively (see Rensink 2000). However, an attended object is not experienced as having any novel feature corresponding to the mere act of focusing the attention. In consequence, it seems that the phenomenal demonstrative aspect does not belong to perceptual phenomenology.

Nevertheless, it is not obvious that the visual phenomenology is restricted to sensory phenomenology presenting properties such as colors, shapes, etc. For instance, Montague proposes that there is also a cognitive phenomenology of perceptual states and this phenomenal demonstrative aspect belongs to such non-sensory perceptual phenomenology (Montague 2011). Similarly, in debates concerning high-level properties perception, authors often postulate types of phenomenology that are associated with perceptual states but cannot be simply identified with sensory phenomenology. For example, Reiland speaks about the phenomenology of perceptual seemings (Reiland 2014), Lyons postulates perceptual judgments (Lyons 2005), and Fish acknowledges the interpretative aspect of perceptual states in addition to the sensory aspect (Fish 2013). 
In the philosophical literature, two types of criteria are usually applied in order to distinguish elements belonging to perceptual phenomenology ${ }^{6}$. The first concerns the influence of background propositional knowledge on the presence of the considered phenomenal element (see Bayne 2009; Block 2014). A second criterion regards the generic of physiological mechanism in virtue of which the phenomenal element occurs (see Lyons 2005; Reiland 2015). Perceptual phenomenology is believed to be largely independent from background knowledge and mainly occurs in virtue of mechanisms that are commonly considered part of a perceptual system.

To see how these criteria work let's consider a case in which one has a visual experience as of a rabbit. Such a person (a) experiences that the object has a certain shape (characteristic of rabbits) and (b) has a belief that the object is a rabbit. Both (a) and (b) plausibly have some phenomenology: it is somehow for a person to see a shape and to form beliefs about rabbits. Nevertheless, there are important differences between (a) and (b). First, experiences as of a shape happen mainly in virtue of perceptual mechanism related to edge detection, perceptual grouping, visual completion, etc. On the other hand, a belief that an object is a rabbit is likely to be produced by postperceptual mechanisms. Second, an experience as of certain shape is largely independent from background knowledge. For instance, one may gain knowledge that in fact the perceived object has a different shape and a person is undergoing some sort of optical illusion. Despite this additional knowledge, the phenomenology of shape experience would not change (in this sense perceptual phenomenology is 'passive', see Reiland 2014). In contrast, in the case of a belief about being

\footnotetext{
${ }^{6}$ In addition, the method of phenomenal contrast, introduced by Siegel (2006), is often referred to in works discussing the perceptual status of some phenomenal changes. However, I treat this method not as a criterion for deciding whether something belongs to perceptual phenomenology, but rather as a method which allows pointing out interesting cases of phenomenal differences which then may be interpreted, by applying some criterion, as involving perceptual or non-perceptual phenomenology.
} 
a rabbit, background knowledge is crucial. If one gains knowledge that the perceived object is not a rabbit, but for instance is a rabbit-hologram, then one will no longer believe that the perceived thing is a rabbit. In consequence, while an experience presenting a shape is likely to have a perceptual phenomenology, the phenomenology of beliefs is probably non-perceptual.

The above reasoning may also be used to demonstrate the presence of non-sensory visual phenomenology. Let's once again consider a case in which a person experiences a thing as having a certain shape, forms a belief that it is a rabbit, but then gains knowledge that the object is a hologram. While the person no longer believes that the object is a rabbit, it still seems to be a rabbit (or it looks as a rabbit). This suggests that there is some sort of visual seeming-phenomenology that is largely independent from background knowledge. In addition, such phenomenology is likely to happen in virtue of high-level perceptual categorization mechanisms that match the experienced shape with a rabbit-prototype stored in long-term memory (see Hummel 2013). In consequence, seeming-phenomenology is plausibly a perceptual phenomenology while not being a sensory perceptual phenomenology.

I believe that the analogous status can be attributed to demonstrative phenomenology related to attentional processing. First, mechanisms allowing for focusing visual attention are without much controversy interpreted as a part of the perceptual system (sometimes such mechanisms are distinguished as constituting 'mid-level vision', see Rensink 2001). Second, the phenomenal change introduced by attentional focus is also - in an important way independent from background knowledge. While the decision concerning a place to which attention is directed is often determined by possessed beliefs (see Yeari and Goldsmith 2010), the demonstrative phenomenology related to attentional processing is not easily modified by gaining propositional data about the attended object. For instance, one may focus attention on an object and as a result have an experience as of this object in place $P$ being red, but later obtain information that there is no red object in $P$ at all, since what one perceives is just a 
mirror-image. Nevertheless, it does not seem likely that this additional piece of knowledge would modify attentional phenomenology such that a person would no longer have an experience as of this red object in $P$.

On these grounds, I believe that there is a perceptual demonstrative phenomenology in the case of visual experiences. In the next section, I argue that such phenomenology may be plausibly interpreted as presenting the specific particularity of objects.

\subsection{Particularity in synchronic and diachronic contexts}

The fact that there is a demonstrative perceptual phenomenology does not by itself entail that this phenomenology presents the specific particularity of attended objects. In fact there are several possible interpretations according to which demonstrative phenomenology has nothing to do with specific particularity. For instance, demonstrative phenomenology may present that the attended object is a subject of perceived properties or that the attended object is a cause of the given experience. In order to plausibly postulate that demonstrative phenomenology presents specific particularity, it has to be shown that it presents something unique for each attended object. One way to satisfy this constraint is to argue that the visual system is equipped with several perceptual demonstratives such that each of them presents an object in a phenomenally distinct and unique way.

Let's start from considering synchronic scenarios in which several objects are experienced at once in a static scene. According to physiological considerations, the human visual system has an ability to represent several, but usually not more than four, objects simultaneously using attentional resources (Scholl 2007). The details differ between psychological models. For instance, relying on the studies concerning object-specific preview benefit, i.e., a phenomenon that a feature is recognized more rapidly if it is presented again on 
the same object, the presence of 'object-files' has been proposed (Kahneman et al. 1992). Each object-file stores information about a distinct object, so they allow the simultaneous representation of several objects. Relying on a different experimental paradigm, known as Multiple Object Tracking, in which participants track several targets among distractors, it has been proposed that vision is equipped with 'visual indices' serving as demonstratives by which the perceptual system refers to objects (Pylyshyn 2007). Furthermore, some authors believe that attentional focus serves as a deictic pointing device (see Ballard 1997) and if visual attention is multifocal (Cavanagh and Alvarez 2005), it can be used in representing several objects at the same time. Despite various differences between models (see Raftopoulos 2009; Vernazzani 2021 for reviews), the postulated representational devices have an important common characteristic: Each of them is used to represent exactly one object, and no two are used to represent the same object. Hence, usage of multiple object files, indices, focuses of attention, or other 'individuation devices' allows the visual system to represent objects as being numerically distinct from each other.

In virtue of such mechanisms, visual perception is able to simultaneously represent several objects as distinct individuals. However, this does not entail that they are phenomenally presented as distinct individuals, or in other words that there are phenomenal presentations of their specific particularity. To obtain this result one should show that usage of individuation devices is associated with some perceptual phenomenal character such that this phenomenal character is distinct for objects represented using a distinct individuation device.

It is commonly acknowledged that the mechanisms responsible for deploying individuation devices are largely attentional (see Green 2018; Scholl 2009). I believe that their attentional status provides a good reason for believing that their use is likely to be associated with perceptual, demonstrative phenomenology. First, as argued in the previous section, the application of significant attentional resources is likely to be connected with 
changes in perceptual phenomenology. This is not to claim that attentional processing is always associated with phenomenal changes. However, attentional processing without phenomenal modifications happens mainly in specific circumstances, especially when presentation of a stimulus is very brief, or stimuli are arranged in such way that the attended stimulus is masked (see van Boxtel et al. 2010; Waztl 2017, 244-248). Such conditions do not occur in the usual situation of applying individuation devices, so it is plausible that related attentional processing leads to modifications of perceptual phenomenology. Furthermore, individuation devices are used to store information about a single entity, refer to a particular object, differentiate one entity from other, and track an individual. In consequence, it seems likely that the phenomenology associated with their use is a demonstrative phenomenology in virtue of which an entity is experienced as this particular object.

Nevertheless, it should still be asked whether, when several individuation devices are used simultaneously, and so several objects are represented, each of them is associated with a distinct demonstrative phenomenology. In contrary, one may propose that by using individuation devices each attended object is presented in phenomenally same way as, let's say, this object distinct from other attended objects.

I believe that in the context of static visual scenes this problem can be resolved by reflecting on the spatial aspect of attention. The phenomenology of focusing visual attention involves deploying it in some direction and at some distance from the subject. In consequence, visual demonstrative phenomenology, at least in the case of normally functioning vision, is not merely this but this at some distance in some direction. Because of this spatial aspect, each of the simultaneously maintained individuation devices is associated with a distinct demonstrative phenomenology. In a static visual scene each pair of objects is usually represented as positioned in distinct directions (i.e., they at most partially overlap) and in rare cases in which they are experienced as positioned in the same direction (e.g., two 
overlapping semi-transparent items), there are some distance cues suggesting which one is farther away (Blaser et al. 2000). As a consequence, each of the simultaneously applied individuation devices phenomenally presents an object in a unique way related to the unrepeatable position of an object in a static visual scene.

The individuation devices used by the human visual system allow us not only to differentiate between objects in a static scene but also to represent objects as being the same despite movement and some qualitative changes (see Scholl 2007). Analogously to static scenes, (a) each individuating device is used in representing only one object and (b) distinct individuating devices represent distinct objects. When contingent changes occur, an object is represented as being the same in virtue of being represented by the same individuating device. In particular, the Multiple Object Tracking experimental paradigm, in which participants track targets among distractors, shows that it is possible to successfully track several randomly moving objects (Pylyshyn 2007). Within vision science, there is a consensus that objects are usually visually represented as being numerically the same as long as their movement is spatiotemporally continuous (von Marle and Scholl 2003). The changes in objects' features, such as color or shape, are less relevant for diachronic identification unless they concern the modification of topological properties (e.g., cutting an object into several pieces, see Zhou et al. 2010).

The approach to phenomenally presented specific particularity in the case of dynamic scenes cannot be the same as in static ones. This is because there is no guarantee that each individuating device will be associated with presenting a unique spatial location. In particular, when an object is experienced as moving, the spatial aspects of demonstrative phenomenology related to a single individuating device are constantly changing. Furthermore, objects may swap their positions or at some point in their movement may overlap. In consequence, there could be an individuating device used in tracking one object that at some 
point is associated with the same spatial phenomenology as an individuating device related to a distinct object.

Despite these additional difficulties, I believe that phenomenal presentations of specific particularity are not restricted to static visual scenes. This is because the demonstrative phenomenology associated with tracking an object has an important aspect involving experiencing an object's spatiotemporal continuity. Such continuity is experienced even if an object changes some features and is briefly occluded, as long as appropriate patterns of spatiotemporal relations are preserved (Hollingworth and Franconeri 2009). A well-known demonstration of phenomenal visual continuity is the so-called "tunnel effect" (see $\mathrm{Xu}$ et al. 2004). When an object is perceived as hiding behind an occluder and as reappearing from the other side after a brief period, people report having an impression that the hiding object is the same one as the appearing object even if their features are different (e.g., the hiding object may be circular while the reappearing one may have a square shape). As long as an object reappears in a place and after a time consistent with the direction and speed of the movement before occlusion, it is perceived as a single object that was moving behind the occluder.

Experiencing spatiotemporal continuity consist in experiencing that an object traverses a series of places such that each place is proximal to the next one. Because of this, demonstrative visual phenomenology in the case of a dynamic scene involves experiencing that this object was at some place $P_{1}$, is currently at some place $P_{2}$ proximal to $P_{1}$, and is moving towards some place $P_{3}$ proximal to $P_{2}$. Such temporal description is consistent with psychological models concerning visual tracking, as they commonly postulate that tracking requires comparing current data with information stored in working memory and that visual mechanisms are engaged in predicting the future position of moving objects (e.g., Atsma et al. 2012). The above phenomenology of continuity allows that in many dynamic scenes each 
object tracked by an individuating device will be phenomenally presented in a unique way. This is because each of the moving objects will be demonstratively experienced as continuously moving through a unique series of places. Even if objects briefly overlap, they will be phenomenally distinct since they previously occupied different places or move forward in different directions. Similarly, when objects swap their positions, each of them may be experienced as moving through the same places but the temporal order of these places will be different in the case of each object.

The above considerations show that human vision has the ability to phenomenally present specific particularity both in static and dynamic scenes. This happens due to the presence of demonstrative spatial phenomenology, which occurs by virtue of the functioning of attentional mechanisms involved in the creation of individuation devices. Such phenomenology presents objects as this object in place $P$ or, in the case of dynamic scenes, as this object moving through places $P_{1}, \ldots, P_{n}$. The demonstrative spatial phenomenology consists of two aspects such that each is necessary for presenting specific particularity. The first is the purely demonstrative aspect that presents this object. By using several individuation devices, the human visual system is able to demonstratively refer to several objects at the same time. While the simultaneous presence of several purely demonstrative phenomenal elements conveys information, given how individuation devices work in the case of human vision, about the number of distinct perceived objects, such purely demonstrative phenomenology does not present what phenomenally differentiates between the experienced objects. This is because each of them is phenomenally presented simply as this object and individuation happens at non-phenomenal level by virtue of utilizing several individuation devices. On the other hand, mere spatial phenomenology without the demonstrative aspect provides only a general description of the surrounding that can be expressed by a quantified proposition such as there is an object $x$ at place $P$ and an object $y$ at place $L$ or, in a dynamic 
context there is an object $x$ at place $P$ at $T_{1}$ and an object $y$ at place $L$ at $T_{2}$. Such a phenomenology does not inform us how many objects there are, as it does not convey information about identity, or distinctiveness, between $x$ and $y$. To present specific particularity, the purely demonstrative aspect has to be combined with a spatial aspect. The demonstrative spatial phenomenology conveys information about the number of distinct objects, in virtue of the demonstrative aspect, and attributes a unique phenomenal characteristic to each of these objects in virtue of the spatial aspect. In consequence, an object can be phenomenally presented as individual particulars distinct from other simultaneously presented objects.

It should be noted that the demonstrative spatial phenomenology cannot be easily replaced by some other kind of demonstrative qualitative phenomenology like demonstrative color phenomenology or demonstrative shape phenomenology. This is because non-spatial characteristics are less likely to differentiate visually presented objects. For instance, while in a static scene no two objects can be experienced as having exactly the same spatial characteristics, many objects may be presented as having the same color and shape. Furthermore, demonstrative spatial phenomenology is more plausible given the way in which visual attention works. Attributing visual attention always involves attending to some spatial region or spatially extended object and in the case of a dynamic scene, attending to an object requires tracking its movement through a spatial trajectory.

However, while human vision has the ability to phenomenally present specific particularity, this ability faces serious restrictions. First, the particularity of only a few objects at a time can be phenomenally presented. Each presentation of specific particularity happens in virtue of a different individuation device and the visual system is only able to simultaneously maintain a few such devices. Second, experiencing specific particularity requires continuous observation. If an object is represented using an individuating device, 
then disappears for a longer period and appears again, information about its earlier appearance may no longer be stored in visual working memory and in consequence the visual system may not be able to represent either the sameness or distinctiveness of the earlier and later objects. In such a situation possible experiences of objects' sameness have to rely on postperceptual data like propositional knowledge about the given object. Third, even if a presentation is continuous, the specific particularity may not be experienced between objects separated by a significant temporal distance. For instance, an object at $T_{1}$ may be experienced as this object at place $P$ and an object at $T_{100}$ may also be experienced as this object at place $P$. However, the visual system may not be able to store data across so many moments and as a result the object at $T_{100}$ cannot be perceptually experienced as related in any way to the object at $T_{1}$. Fourth, specific particularity cannot be presented if something prevents the visual system from establishing an individuation device for a given object. For example, a scene may be too crowded, objects may move too fast, or attentional resources may be unavailable due to being used in a different task.

The presented approach accommodates an intuition that one can, in principle, introspectively recognize differences between phenomenally distinct experiences. In particular, distinct phenomenal presentations of specific particularity can be introspectively distinguished by recognizing differences in the number of objects attended to (in virtue of the demonstrative aspect) and differences in features that are unique to each of the objects attended to (in virtue of the spatial aspect). Furthermore, the above approach to experiencing specific particularity does not exclude the possibility of incorrect experiences of objects' individuality. In particular, in case of a dynamic scene, when two tracked objects move close to each other such that their trajectories are similar, it sometimes happens that their identities are swapped (see Drew et al. 2013). For instance, if at some moment $T_{x}$ object $A$ moves close to object $B$, it may happen that after this moment $A$ will be experienced as continuous with $B$ 
and vice versa. In such a case two specific particularities are experienced, but they are experienced inaccurately. This is because object $A$ is experienced as being the same with the object that previously has been in the position in fact occupied by object $B$ and conversely, object $B$ is experienced as coming from the place that was actually occupied by object $A$.

\section{Conclusions}

I argue that human vision is not able to phenomenally present generic particularity of objects but that it has some ability to present specific particularity. In the case of generic particularity, considerations regarding the rules of visual perceptual organization only allow justification of a weaker thesis - that vision, under normal conditions, does not present characteristics incompatible with generic particularity, or that vision sometimes presents characteristics which entail generic particularity. The ability to experience specific particularity is possessed in virtue of a demonstrative spatial phenomenology associated with the application of visual individuating devices. However, such abilities are severely restricted, as the specific particularity of only a limited number of objects can be experienced at the same time, and visual mechanisms cannot establish an object's sameness across longer periods.

\section{Acknowledgements}

The author would like to thank the anonymous reviewer for comments concerning the paper. The early version of the paper was presented during SemDok seminar at Jagiellonian University.

The work was supported by the National Science Center (Poland) grant 2016/20/S/HS1/00090. 


\section{References}

Almäng, J. 2013. The causal self-referential theory of perception revisited. Dialectica 67(1): $29-53$.

Armstrong, D. M. 1978. Universals and scientific realism. Vol. II: A theory of universals. Cambridge: Cambridge University Press.

Armstrong, D. M. 1997. A world of states of affairs. Cambridge: Cambridge University Press,

Atsma, J., A. Koning, and R. van Lier. 2012. Multiple object tracking: Anticipatory attention doesn't "bounce”. Journal of Vision 12(13): 1-11.

Ballard, D. H., M. M. Hayhoe, P. K. Pook, and R. P. N. Rao 1997. Deictic codes for the embodiment of cognition. Behavioral and Brain Sciences 20: 723-67.

Bayne, T. 2009. Perception and the reach of phenomenal content. The Philosophical Quarterly 59(236): 385-404.

Baxter, D. L. M. 2001. Instantiation as partial identity. Australasian Journal of Philosophy, 79(4), 449-464.

Beck, O. 2019. Two conceptions of phenomenology. Philosophers' Imprint 19(37): 1-17.

Biederman, I. 1987. Recognition-by-components: A theory of human image understanding. Psychological Review 94(2): 115-47.

Blaser, E., Z. W. Pylyshyn, and A.O. Holcombe 2000. Tracking an object through feature space. Nature, 408(6809): 196-9.

Block, N. 2014. Seeing-as in the light of vision science. Philosophy and Phenomenological Research 89(1): 560-72.

Brewer, B. 2011. Perception and its objects. Oxford: Oxford University Press.

Brewer, B. 2015. The object view of perception. Topoi 36(2): 215-27.

Brown, S. 2017. Against instantiation as identity. Philosophical Studies, 174, 887-900. 
van Boxtel, J. J. A., N. Tsuchiya, and C. Koch 2010. Consciousness and attention: On sufficiency and necessity. Frontiers in Psychology 1/217, doi:10.3389/fpsyg.2010.00217.

Byrne, A. 2001. Intentionalism defended. Philosophical Review 110(2): 199-240.

Campbell, J. 2002. Reference and consciousness. Oxford: Oxford University Press.

Carrasco, M., and A. Barbot 2019. Attention alters appearance. Current Opinion in Psychology 29: 56-64.

Cavanagh, P., and A. A. Alvarez 2005. Tracking multiple targets with multifocal attention. Trends in Cognitive Science 9(7): 349-54.

Cowling, S. 2014. Instantiation as location. Philosophical Studies 167: 667-82.

Daly, C. 1994. Tropes. Proceedings of the Aristotelian Society 94: 253-61.

Dokic, J. 1998. The ontology of perception: bipolarity and content. Erkenntnis 48: 153-69

Drew, T., T. S. Horowitz, and E. K. Vogel 2013. Swapping or dropping? Electrophysiological measures of difficulty during multiple object tracking. Cognition 126: 213-23.

Ehring, D. 2004. Distinguishing universals from particulars. Analysis 64(4): 326-32.

Ehring, D. 2011. Tropes, properties, objects, and mental causation. Oxford: Oxford University Press.

Elder, J. H., and R. M. Goldberg 2002. Ecological statistics of gestalt laws for the perceptual organization of contours. Journal of Vision 2(4): 324-53.

Fish, W. 2013. High-level properties and visual experience. Philosophical Studies 162: 4355.

Fuller, S., and M. Carrasco 2006. Exogenous attention and color perception: Performance and appearance of saturation and hue. Vision Research 46: 4032-47.

Giberman, D. 2016. Indiscernibility does not distinguish particularity. Thought: A Journal of Philosophy 5(4): 249-56. 
Gomes, A., and C. French 2016. On the particularity of experience. Philosophical Studies 173: 451-60.

Gomes, A., and C. French 2019. How naïve realism can explain both the particularity and the generality of experience. Philosophical Quarterly 69(274): 41-63.

Green, E. J. 2016. Representationalism and perceptual organization. Philosophical Topics 44(2): $121-48$.

Green, E. J. 2018. What do object files pick out?. Philosophy of Science 85(2): 177-200.

Heil, J. 2003. From an ontological point of view. Oxford: Oxford University Press.

Hill, C. S. 2019. Perceptual existentialism sustained. Erkenntnis, https://doi.org/10.1007/s10670-019-00160-z.

Hollingworth, A., and S. L. Franconeri 2009. Object correspondence across brief occlusion is established on the basis of both spatiotemporal and surface feature cues. Cognition 113: 15066.

Hummel, J. E. 2013. Object recognition. In Oxford Handbook of Cognitive Psychology, ed. D. Reisburgm, 32-46. Oxford: Oxford University Press.

Jeshion, R. 2010. Singular thought: Acquaintance, semantic instrumentalism, and cognitivism. In New essays on singular thought, ed. R. Jeshion, 105-40. Oxford: Oxford University Press.

Kahneman, D., A. M. Treisman, and B. J. Gibbs 1992. The reviewing of object files: Objectspecific integration of information. Cognitive Psychology 24(2): 175-219.

Klatzky, R. L. 1998. Allocentric and egocentric spatial representations: Definitions, distinctions, and interconnections. In Spatial cognition. Lecture notes in computer science, eds. C. Freksa, C. Habel, and K. F. Wender, 1-17. Berlin: Springer.

Kubovy, M., A. O. Holcombe, and J. Wagemans 1998. On the lawfulness of grouping by proximity. Cognitive Psychology 35(1): 71-98. 
Kubovy, M., and J. Wagemans 1995. Grouping by proximity and multistability in dot lattices: A quantitative gestalt theory. Psychological Science 6(4): 225-34.

Logue, H. 2012. Why naive realism?. Proceedings of the Aristotelian Society, 112: 211-37.

Loux, M. J. 1978. Substance and attribute. A study in ontology. Dordrecht: D. Reidel.

Lowe, E. J. 2006. Four-category ontology. A metaphysical foundations for natural sciences. Clarendon Press: Oxford.

Lyons, J. 2005. Perceptual beliefs and nonexperiential looks. Philosophical Perspectives 19: $237-56$.

MacBride, F. 1998. Where are particulars and universals?. Dialectica 52(3): 203-27.

Macpherson, F. 2006. Ambiguous figures and the content of experience. Nous 40(1): 82-117. van Marle, K., and B. J. Scholl 2003. Attentive tracking of objects versus substances. Psychological Science 14(5): 498-504.

Martin, M. 1992. Sight and touch. In The contents of experience, ed. T. Crane, 196-215. Cambridge: Cambridge University Press.

Martin, M. G. F. 2002a. Particular thought and singular thoughts. Royal Institute of Philosophy Supplement 51: 173-214.

Martin, M. G. F. 2002b. The transparency of experience. Mind \& Language 17(4): 376-425.

Melia, J. 2008. A world of concrete particulars. Oxford Studies in Metaphysics 4: 99-124.

Mehta, N. 2014. The limited role of particulars in phenomenal experience. Journal of Philosophy 111(6): 311-31.

Montague, M. 2011. The phenomenology of particularity. In Cognitive phenomenology, eds.

T. Bayne and M. Montague, 121-40. Oxford: Oxford Univeristy Press.

Moreland, J. P. 2001. Universals. Chesham: Acumen.

Nanay, B. 2012. Perceiving tropes. Erkenntnis 77: 1-14. 
O’Callaghan, C. 2008. Object perception: Vision and audition. Philosophy Compass 3(4): 803-29.

Orilia, F., and M. P. Paoletti 2020. Properties. The Stanford Encyclopedia of Philosophy $\begin{array}{llllll}\text { (Winter } & 2020 & \text { Edition), } & \text { Edward } & \text { N. } & \text { Zalta }\end{array}$ https://plato.stanford.edu/archives/win2020/entries/properties/.

Palmer, S., and I. Rock (1994). Rethinking perceptual organization: The role of uniform connectedness. Psychonomic Bulletin and Review 1(1): 29-55.

Prinzmetal, W., H. Amiri, K. Allen, and T. Edwards 1998. Phenomenology of attention: 1. Color, location, orientation, and spatial frequency. Journal of Experimental Psychology: Human Perception and Performance 24(1): 261-82.

Pylyshyn, Z. W. 2007. Things and places. Cambridge, MA: MIT Press.

Qiu, F. T., and R. von der Heydt 2005. Figure and ground in the visual cortex: V2 Combines stereoscopic cues with gestalt rules. Neuron 47(1): 155-66.

Raftopoulos, A. 2009. Cognition and Perception. How Do Psychology and Neural Science Inform Philosophy?. Cambridge, MA: The MIT Press.

Reiland, I. 2014. On experiencing high-level properties. American Philosophical Quarterly 51: $177-87$.

Reiland, I. 2015. Experience, seemings, and evidence. Pacific Philosophical Quarterly 96: $510-34$.

Rensink, R. A. 2000. The dynamic representation of scenes. Visual Cognition 7(1/2/3): 1742.

Rensink, R. A. 2001. Change blindness: Implications for the nature of visual attention. In Vision and attention, eds. M. Jenkin and L. Harris, 169-88. New York: Springer.

Schellenberg, S. 2010. The particularity and phenomenology of perceptual experiences. Philosophical Studies 149: 19-48. 
Schellenberg, S. 2016. Perceptual particularity. Philosophy and Phenomenological Research 93(1): 25-54.

Scholl, B. J. 2007. Object persistence in philosophy and psychology. Mind \& Language 22(5): 563-91.

Scholl, B. J. 2009. What have we learned about attention from multiple-object tracking (and vice versa)?. In Computation, cognition, and Pylyshyn, eds. D. Dedrick and L. Trick, 49-77. Cambridge, MA: The MIT Press.

Schroer, R. 2014. The goldilocks problem and the specificity of visual phenomenal content. Canadian Journal of Philosophy, 44(3-4), 476-95.

Skrzypulec, B. 2019. Olfactory objecthood. Philosophia 47(3): 881-900.

Soteriou, M. 2000. The particularity of visual perception. European Journal of Philosophy 8(2): 173-89.

Tye, M. 1995. Ten problems of consciousness. Cambridge, MA: MIT Press.

Vernazzani, A. 2021. Do we see facts? Mind \& Language, https://doi.org/10.1111/mila.12336.

Watzl, S. 2017. Structuring mind. The nature of attention and how it shapes consciousness. Oxford: Oxford University Press.

Yeari, M., and M. Goldsmith 2010. Is object-based attention mandatory? strategic control over mode of attention. Journal of Experimental Psychology: Human Perception and Performance 36(3): 565-79.

Xu F., S. Carey, and N. Quint 2004. The emergence of kind-based object individuation in infancy. Cognitive Psychology 49(2): 155-90.

$\mathrm{Xu}, \mathrm{Y}$., and M. Singh 2002. Early computation of part structure: evidence from visual search. Perception and Psychophysics 64(7): 1039-54. 
Zhou, K., H. Luo, T. Zhou, Y. Zhou, and L. Chen 2010. Topological change disturbs object continuity in attentive tracking. PNAS 107(50): 21920-24. 